\title{
Uso oportuno de inmunoglobulinas intravenosas y riesgo de alteraciones coronarias en pacientes con Enfermedad de Kawasaki.
}

\author{
Nathan Pulido ${ }^{2-3}$, María Eugenia Consigliere ${ }^{2}$, Sebastián Mela ${ }^{1}$, Juan Carlos Caroca ${ }^{1}$, \\ Astrid Sielfeld ${ }^{1}$, Luis Bofill' \\ (1) Interno de Medicina, Universidad Andrés Bello, Viña del Mar. \\ (2) Cardiólogo Infantil, Hospital Dr. Gustavo Fricke, Viña del Mar. \\ (3) Docente de Pediatría, Universidad Andrés Bello, Viña del Mar. \\ (4) Becado de Pediatría, Universidad de Valparaíso, Viña del Mar.
}

Antecedentes: La enfermedad de Kawasaki es una vasculitis sistémica de vasos de mediano calibre, que afecta principalmente a las arterias coronarias, provocando dilatación y aneurismas coronarios en un 15 - $25 \%$ de los casos no tratados. El tratamiento recomendado actualmente es el uso de inmunoglobulina intravenosa antes del décimo día de enfermedad, lo cual reduce el riesgo de aneurismas coronarios de un $20 \%$ a un $4 \%$.

Objetivos: Determinar la relación entre el tratamiento oportuno con inmunoglobulina y el riesgo de presentar alteración coronaria en la ecocardiografía en pacientes con Enfermedad de Kawasaki.

Métodos: Se revisó la base de datos de Cardiología Infantil del hospital, identificando 66 pacientes que pre- sentaron la enfermedad entre los años 1994 y 2014. Se determinó el momento en que se inició el tratamiento con inmunoglobulina y se analizó el hallazgo ecocardiográfico de alteraciones coronarias durante el período de hospitalización.

Resultados: 52 niños (79\%) recibieron tratamiento precoz, antes de 10 días del comienzo de la enfermedad; en el resto el tratamiento fue tardío. El tratamiento precoz se asoció a un menor riesgo de presentar aneurismas coronarios (5.8\% vs $50 \%$ de los casos, $\mathrm{p}<0.01)$.

Conclusión: En la enfermedad de Kawasaki es de suma importancia el diagnóstico y tratamiento oportuno con inmunoglobulina intravenosa para reducir el riesgo de formación de aneurismas coronarios.

Correspondencia:

Sebastián Mela S.

smela.sfeir@gmail.com 


\section{Early use of immunoglobulin and risk of coronary abnormalities in patients with Kawasaki's disease}

Background: Kawasaki's disease is a systemic vasculitis of the medium-sized blood vessels, primarily affecting the coronary arteries, causing coronary dilation and aneurysms in $15-25 \%$ of untreated children. Use of Intravenous immunoglobulin within the first 10 days of illness is the recommended treatment, redugin the risk of coronary artery aneurysm development from $20 \%$ to $4 \%$.

Objective: To determine the association of early treatment to risk of echocardiographic coronary changes on patients with the disease.

Methods and Results: A review of the institutional Pediatric Cardiology Database, allowed the identification of 66 patients who had Kawasa- ki's disease between 1994 and 2014. The echocardiographic findings at follow up were related to the time of treatment initiation in relation to the beginning of the disease. Treatment with immunoglobulin within 10 days was associated to coronary artery aneurysms in $5.8 \%$ of patients, as opposed to $50 \%$ in those with a delayed initiation of therapy.

Conclusion: Early treatment with immunoglobulins (within 10 days of illness) significantly reduces the short term risk of developing coronary aneurysms in patients with Kawasaki's disease.

Key words: Kawasaki's disease, coronary aneurysm, immunoglobulin. 
Introducción: La Enfermedad de Kawasaki (EK) es una entidad clínica poco frecuente y representa la principal causa de cardiopatía adquirida en niños. Corresponde a una vasculitis sistémica de causa desconocida, afectando principalmente arterias de mediano calibre. Estos cambios vasculares inflamatorios agudos pueden evolucionar a compromiso coronario, miocarditis y pericarditis, entre otros. La afección central y más relevante ocurre en las arterias coronarias por inflamación de la capa media, con edema y necrosis del músculo liso, favoreciendo la dilatación y formación de aneurismas. ${ }^{1-2}$ En etapas más avanzadas puede desarrollarse estenosis y oclusión arterial por trombosis sobre-agregada, llevando a infarto agudo al miocardio y potencial muerte súbita. ${ }^{3}$ La incidencia tiene grandes variaciones geográficas y étnicas. En Japón, país con la mayor incidencia, se observan 90 casos por 100.000 personas al año, mientras que en Chile alcanza entre 3-4 casos por 100.000 personas al año ${ }^{4}$. Se estima la mortalidad mundial entre 0,1 y $3 \% .^{4}$. La población afectada se agrupa principalmente entre los 3 meses y los 5 años de edad, donde se concentra más del $80 \%$ de los casos. La relación entre hombres y mujeres es de 7:5.4

El diagnóstico se realiza clínicamente, con la presencia de fiebre de 5 o más días de duración, acompañado de 4 de 5 de los siguientes signos: conjuntivitis bilateral no exudativa; ras polimorfo; cambios de mucosa oral (labios eritematosos, fisurados, secos, lengua aframbuesada, mucosa oral y faríngea eritematosa); cambios de extremidades (eritema palmo plantar, edema y/o descamación de manos y pies); y adenopatía cervical única > $15 \mathrm{~mm}$, dolorosa, no supurada. ${ }^{6}$ Se denomina "Atípica" o "Incompleta" cuando se cumplen solo 3 de los 5 signos (aproximadamente el 10\% de los casos). . $^{5-6}$

Las guías clínicas actuales recomiendan el uso de Inmunoglobulina intravenosa como tratamiento (IGIV) en dosis de $2 \mathrm{~g} / \mathrm{Kg}$, administrada preferentemente entre el quinto y décimo día de enfermedad (desde que se inicia la fiebre), y el uso de ácido acetilsalicílico (AAS). Esto reduce el riesgo de formación de aneurismas coronarios de un $20 \%$ a un $4 \%$ en comparación a pacientes no tratados. El uso de AAS es principalmente como antiinflamatorio en la fase aguda, a una dosis equivalente a $80-100$ $\mathrm{mg} / \mathrm{kg} /$ día fraccionado cada 6 horas, hasta el cuarto día afebril, para luego bajar la dosis a efecto anti-plaquetario (3-5 mg/kg/día) durante 6 a 8 semanas. . $^{3-7}$

La dilatación coronaria puede detectarse a partir de los 7-10 días de iniciada la enfermedad, y alcanza su máxima incidencia en una etapa subaguda, durante la tercera y cuarta semana. ${ }^{8}$

La Guía 2004 del American Heart Association describe el uso de un z-score para la determinación de alteraciones coronarias, relacionado a la superficie corporal del niño. La técnica requiere de la medición del diámetro interno del vaso, por un ecografista con experiencia pediátrica. Se define como normal un valor $\mathrm{z}$ de $+/-2$ en relación con niños sanos de similar superficie corporal. Una dilatación o ectasia coronaria se define como una medida $\mathrm{z}>2$ DS o con un diámetro $>$ o igual a $2 \mathrm{~mm}$, en forma difusa. Aneurisma coronario se define como una dilatación localizada con $\mathrm{z}$ valor $\mathrm{z}>2$ DS y aneurisma gigante si mide más de $8 \mathrm{~mm}$ de diámetro (1-4\% de los pacientes)..$^{3-9-10}$ En el seguimiento a largo plazo de los pacientes se ha observado que el retroceso de las anomalías coronarias ocurre hasta en 50\% de los casos. Sin embargo, las arterias permanecen histológica y funcionalmente alteradas, lo cual lleva a una progresiva estenosis. Se estima que un $40 \%$ de los pacientes con aneurismas coronarios persistentes y estenosis coronaria desarrollan infarto del miocardio. ${ }^{3}$

El presente trabajo tiene como objetivo determinar la relación entre el tratamiento oportuno con Inmunoglobulinas Intravenosas y el riesgo de presentar alteración coronaria en la ecografía cardiovascular en pacientes pediátricos con Enfermedad de Kawasaki.

Métodos: La presente investigación corresponde a un estudio descriptivo, retrospectivo, de serie de casos. Los registros analizados en esta investigación fueron obtenidos a través de la revisión sistemática de la base de datos de pacientes que padecieron Enfermedad de Kawasaki, propiedad del servicio de pediatría y cardiología infantil del hospital.

Se analizaron niños con diagnóstico de enfermedad de Kawasaki, entre 4 meses y 8 años de edad, de ambos sexos, atendidos en el Servicio de Pediatría del Hospital Dr. Gustavo Fricke entre enero de 1994 y octubre de 2014. Además, requerían haber sido tratados con IGIV en dosis de $2 \mathrm{~g} / \mathrm{Kg}$ y que se les hubiera realizado una ecografía cardiovascular con medición de arterias coronarias durante la hospitalización. De los 69 pacientes con EK durante este período fueron incluidos 66. Dos pacientes no fueron considerados por no haber sido tratados con IGIV, y otro paciente fue excluido por tener datos incompletos en la base de datos.

El análisis posterior consistió en determinar la relación entre el tratamiento con IGIV antes o después de los primeros 10 días de enfermedad y la alteración coronaria 


\begin{tabular}{|c|r|r|r|}
\hline \multicolumn{3}{|c|}{ Tabla 1. Características clínicas en ambos grupos de pacientes con } \\
Enfermedad de Kawasaki
\end{tabular}

evidenciada en el ecocardiograma. Se consideraron 4 categorías para determinar la medida del diámetro de arterias coronarias, ajustando cada medida con la superficie corporal del niño: normal $(<2 \mathrm{~mm})$, dilatación difusa $(2$ $\mathrm{mm}-<4 \mathrm{~mm})$, aneurisma $(4 \mathrm{~mm}-<8 \mathrm{~mm})$ y aneurisma gigante $(>8 \mathrm{~mm})$.

Los datos obtenidos se analizaron con el programa SPSS Statistics ${ }^{20}$. Se realizó una prueba de independencia con chi2 para evaluar la relación entre las alteraciones coronarias y el momento del tratamiento.

Resultados: De los 66 pacientes estudiados, 52 $(78,8 \%)$ recibieron un tratamiento de manera precoz, y $14(21,2 \%)$ lo hicieron de forma tardía. Del total de pacientes, 62 (94\%) tenía entre 4 meses y 5 años de edad. La relación hombre/mujer fue de 1,37:1. La gran mayoría de los pacientes cumplieron con 4 de los 5 criterios clínicos para el diagnóstico de la enfermedad; solo las adenopatías cervicales estuvieron presentes en un número cercano al 50\% (Tabla 1).

Siguiendo la experiencia internacional, todos los pacientes estaban programados para recibir inmunoglobulina desde el ingreso. Las ecografías se efectuaron durante la hospitalización.

Los resultados de la ecocardiografía con mediciones de arterias coronarias, mostraron que 34 pacientes $(51,5 \%)$ presentaron mediciones normales ( $<2 \mathrm{~mm}$ de diámetro), $19(28,8 \%)$ presentaron dilatación coronaria $(2 \mathrm{~mm}-<4$ $\mathrm{mm})$ y $10(15,2 \%)$ presentaron aneurismas $(4-<8 \mathrm{~mm})$. No hubo hallazgos de aneurismas gigantes en la muestra estudiada. Por otro lado, 3 pacientes $(4,5 \%)$ no tuvieron alteraciones coronarias, pero sí presentaron insuficiencia cardíaca o anormalidades en la ecografía como pericarditis o miocarditis e insuficiencia cardíaca.

De los 52 pacientes que recibieron tratamiento precoz con IGIV, $31(59,6 \%)$ tuvieron ecografía normal, 15 (28,8\%) con dilatación coronaria, $3(5,8 \%)$ con aneurisma coronario y $3(5,8 \%)$ con otros hallazgos cardíacos patológicos no coronarios. Mientras que de los 14 pacientes que recibieron tratamiento tardío con IGIV, $3(21,4 \%)$ presentaron ecografía normal, $4(28,6 \%)$ con dilatación coronaria y $7(50 \%)$ con aneurisma coronario ( $p<0,001)$, (Tabla 2$)$.

\section{Discusión}

Como se puede apreciar en los resultados, el tratamiento precoz con IGIV en pacientes con EK está asociado significativamente a un menor riesgo de presentar aneurismas coronarios en comparación con los que recibieron

Tabla 2. Alteraciones ecocardiográficas coronarias observadas en pacientes con Enfermedad de Kawasaki tratados precoz o tardíamente con immunoglobulina

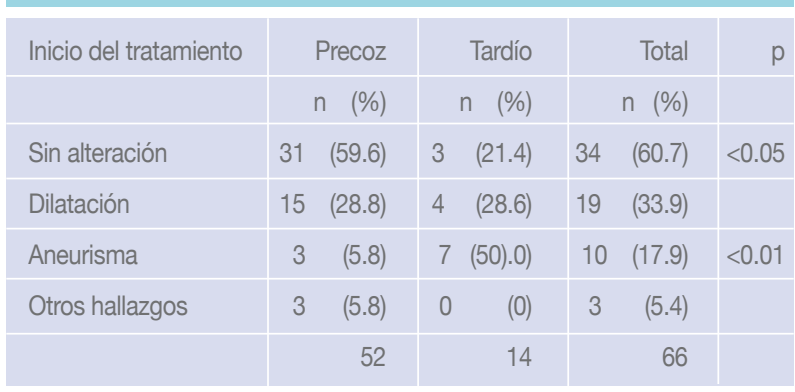


un tratamiento posterior al décimo día (5.8\% vs 50\%). Esto concuerda con la literatura actual, que estima la presencia de aneurismas coronarios en alrededor del $4 \%$ de los pacientes que recibieron un tratamiento oportuno ${ }^{3}$. Además, un tratamiento oportuno se asoció a una probabilidad tres veces mayor de presentar una ecografía normal. Este resultado resalta la importancia de entregar un tratamiento precoz frente a un paciente con EK, para disminuir de esta manera el riesgo coronario.

El presente estudio presenta limitación que deben destacarse. La principal consiste en que la selección de los grupos comparados no es aleatoria. En efecto, el tratamiento tardío no fue determinado por los investigadores sino una consecuencia del tiempo de llegada del paciente al hospital. En este sentido, la comparación es retrospectiva pero el objetivo primario no era evaluar la efectividad del tratamiento sin las consecuencias de su iniciación tardía sobre el desarrollo de las alteraciones anatómicas de la aorta y, en especial, la formación de aneurismas coronarios. Una segunda limitación es que el seguimiento de los pacientes no fue planificado en forma prospectiva y la información dependió de los datos de la ficha clínica y de la base de datos. Sin embargo, estas limitaciones no son determinantes para invalidar las conclusiones, especialmente el efecto protector del tratamiento precoz con inmunoglobulinas para prevenir la formación de aneurismas coronarios.

En conclusión, es muy importante el diagnóstico oportuno de la Enfermedad de Kawasaki y derivar los pacientes precozmente a un centro que cuente con la capacidad de un tratamiento precoz con inmunoglobulinas EV. Así se logrará disminuir el riesgo de presentar alteraciones coronarias, sobre todo aneurismas coronarios, como también reducir las probabilidades de complicaciones cardiovasculares futuras.

Agradecimientos: Al Dr. Tomás Montecinos, Cardiólogo infantil del Hospital Dr. Carlos Van Buren y Docente de UNAB Viña del Mar, por su apoyo y recomendaciones en la ejecición del trabajo. 


\section{Referencias:}

1. RIZK SR, EL SAID G, DANIELS LB, BURNS JC, EL SAID $\mathrm{H}$, SOROUR KA, et al. Acute myocardial ischemia in adults secondary to missed Kawasaki disease in childhood. JACC, 2014.

2. HOLVE TJ, PATEL A, CHAU Q, MARKS AR, MEADOWS A, ZAROFF JG. Long-term Cardiovascular Outcomes in Survivors of Kawasaki Disease. PEDIATRICS 2014; 133: e305-11.

3. PREGO J. Enfermedad de Kawasaki, puesta al día. Arch Pediatr Urug 2003; 74: 99-113.

4. BUDNIK OI, HIRSCH BT, FERNÁNDEZ CC, YÁNEZ PL, ZAMORANO R J. Enfermedad de Kawasaki: una serie clínica. Rev Chil Infect 2011; 28: 416-422.

5. SUNDEL R. Kawasaki disease: Clinical features and diagnosis. En: UpToDate (Revisado en Noviembre, 2014.).

6. AYUSAWA M. Revision of diagnostic guidelines for Kawasaki disease (the 5th revised edition). Pediatrics International. 2005; 47: 232-234.

7. YELLEN ES, GAUVREAU K, TAKAHASHI M, BURNS JC, SHULMAN S, ET AL. Performance of 2004 American Heart Association Recommendations for Treatment of Kawasaki Disease. PEDIATRICS. 2010; 125: e234-41.

8. TSE S, SILVERMAN E, MCCRINDLE B, YEUNG R. Early treatment with intravenous immunoglobulin in patients with Kawasaki disease. J Pediatr 2002; 140: 450.

9. SELAMET TIERNEY ES, GAL D, GAUVREAU K, BAKER AL, TREVEY S, O'NEILL SR, et al. Vascular Health in Kawasaki Disease, Journal of the American College of Cardiology, 2013; 17; 62: 1114-21. Published by Elsevier Inc: 1114-21.

10. YELLEN E, GAUVREAU K, TAKAHASHI M, et al. Performance of 2004 American Heart Association recommendation for treatment of Kawasaki disease, Pediatrics 2010; 125: e234-41. 